\title{
EXPECTATIONS FOR ASTROMETRY WITH THE HUBBLE SPACE TELESCOPE
}

\author{
R.L. Duncombe \\ Center For Space Research \\ University of Texas at Austin \\ Austin, TX 78712 \\ and \\ W.H. JefFerys, G.F. Benedict, P.D. Hemenway and P.J. Shelus \\ Department of Astronomy \\ University of Texas at Austin \\ Austin, TX 78712
}

\begin{abstract}
The Hubble Space Telescope, a large optical instrument having an aperture of 2.4 meters and a length of 8.8 meters has been developed by the U.S. National Aeronautics and Space Administration in cooperation with the European Space Agency. The Space Shuttle will be used to place the telescope in orbit. The primary astrometric instrument will be one of the three Fine Guidance Sensors which have the capability of measuring the position of one object with respect to another to an accuracy of \pm 0.002 . To facilitate use of the Hubble Space Telescope, observers will be provided with the Astrometric Data Reduction Software package. The variety of astrometric problems and the several modes of operation are mentioned as well as the cooperative program with the European astrometric satellite project HIPPARCOS.
\end{abstract}

\section{Hubble Space Telescope Astrometry}

The Hubble Space Telescope (HST) will have the capability to measure the relative positions of stars within the field of view of a Fine Guidance Sensor (FGS) with an accuracy of $\pm 0.002 \mathrm{rms}$. The magnitude range of HST astrometry using an FGS is from 9 to 17, which may be extended to 4th magnitude with the use of filters. While two FGSs are used to control the pointing of the HST, the third FGS may be used for astrometric measurements. FGS \#2 has been designated as the prime FGS for astrometry because of guidance considerations.

Each FGS views a ninety degree sector of an annulus which comprises the outer portion of the HST focal plane (Figure 1). Each sector of the annulus (hereafter referred to as a "pickle") is four arcminutes wide and has a maximum chord length of about eighteen arcminutes. The measurement accuracy quoted above refers to the relative positions of two objects within the central twenty square arcminutes of the same pickle.

Figure 2 is a schematic diagram of an FGS. Each FGS includes an optical beam splitter, two orthogonal Koester's prism interferometers (one for each axis), and their associated photomultiplier tubes. Light from an object anywhere in the pickle is brought into the five arcsecond square aperture 


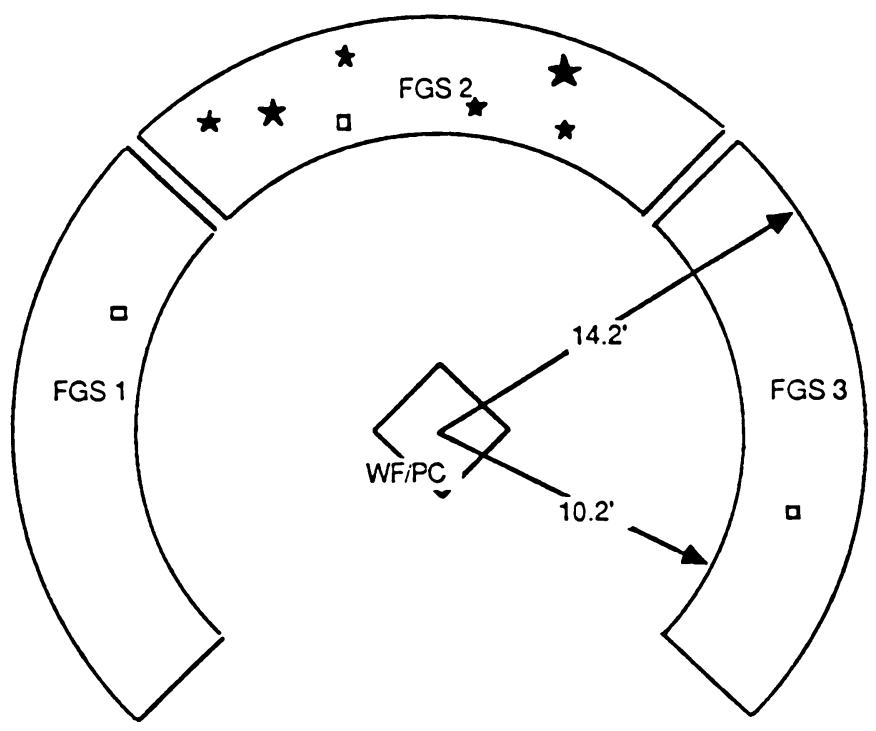

Figure 1. HST focal plane, with locations of FGS fields of view.

of the FGS detector assembly by the rotation of two beam deflectors called "star selectors." If the object is directly on the axis of an interferometer, the signal from each of the two photomultipliers associated with that axis will be equal, and their difference will be zero. If the object is off the axis, the difference of the two photomultiplier readings will provide an error signal that indicates how far off-axis the object is. FGS circuitry then nulls the error signal by repositioning the star selectors to bring the object back onto the interferometer axis. The position of one object with respect to another in the same pickle can be calculated from the angles $\theta_{A}, \theta_{B}$ and $\Delta \theta_{A}, \Delta \theta_{B}$ produced by the star selector positions as shown in Figure 3.

The FGS has several modes of operation, when used as an astrometric instrument. In the "lockon" mode, the interferometers are nulled successively on each object being measured within the same pickle. In the "multiple star" mode, the FGS aperture is moved diagonally across the target. The error signal in each axis varies as the aperture moves across the target, tracing out the instrumental transfer function (Figure 4). The transfer function can then be analyzed to detect duplicity of stars and to measure the relative positions of the two components. In the "moving target" mode, which is used for tracking targets such as asteroids, the FGS is kept locked on the object and its position is recorded periodically.

The "transit circle" mode will be used to observe objects fainter than the 17th magnitude. In this mode the Wide Field Planetary Camera (WFPC) will be used in conjunction with the two guiding 


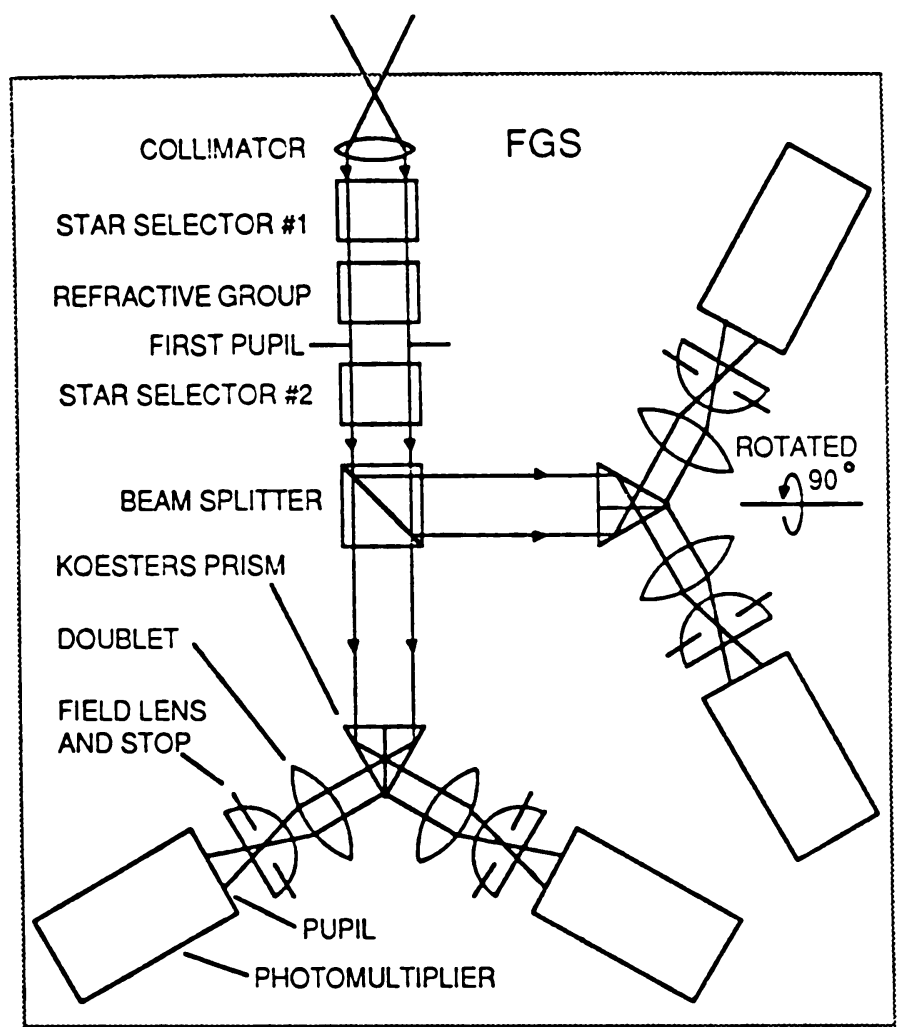

Figure 2. Schematic diagram of the FGS.

FGS units. The WFPC will be used to observe the two faint target objects in turn, while the HST is positioned so that the same pair of guide stars can be used for guiding during both observations. The position of the first faint object is measured with respect to the WFPC itself. Then the HST is repositioned to measure the second object with respect to the WFPC. The relative positions of the two target objects is the difference between the centroided images in the WFPC frame plus the difference between the FGS pointings on the same pair of guide stars. These observations are expected to produce results only slightly less accurate than those made by the astrometric FGS alone.

It can be seen from the foregoing that there are some restrictions on the use of an FGS for astrometry. Since two of the FGS are required for HST guidance, only one FGS is available for astrometric observations. Further, the FGS is not an imaging instrument; it can not see an object but can only sense its presence after moving to the object's approximate location. For this reason the 


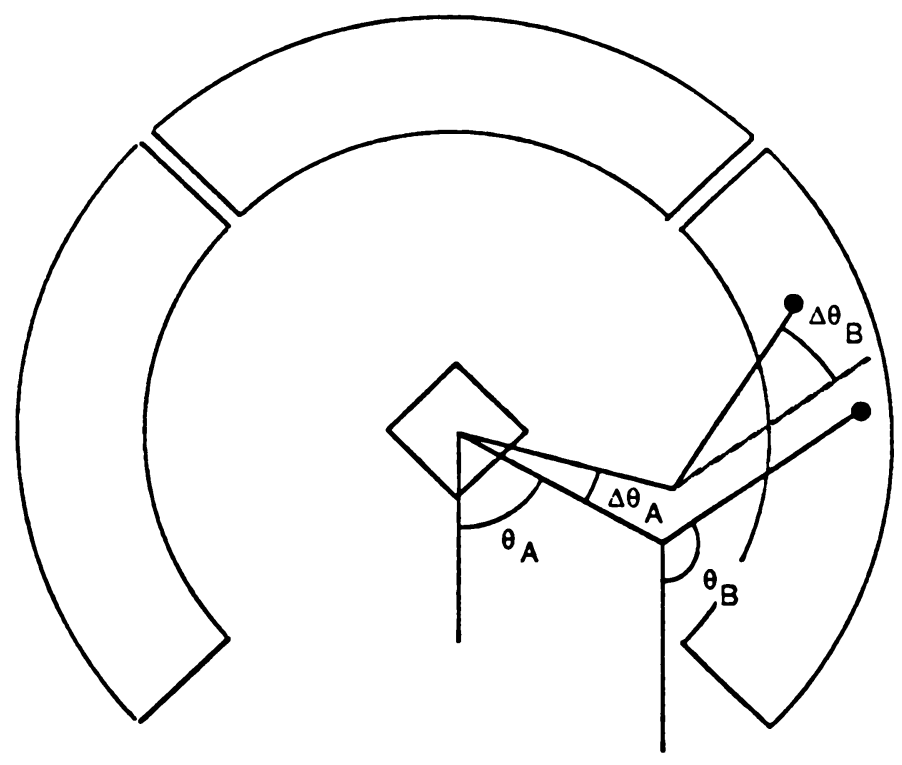

Figure 3. Geometry of the star selector coordinates.

pickle area must be inventoried in advance, and the successive observations pre-planned in order to direct the FGS aperture to each target in turn.

The ultimate determination of HST capabilities and limitations for astrometry in space will be made during the periods of Orbital Verification and Science Verification following launch. A star field, well-calibrated by ground observations, will be used to determine the zero-order plate scale and field distortion of the FGS. Since no star field is available which contains relative positions accurate to $\pm 0: 002$ over a range of 18 arcminutes, an overlapping plate technique will be used in conjunction with the Optical Field Angle Distortion (OFAD) program. Then a moving target, such as an asteroid, will be tracked across an FGS field to measure the scale factor within the measuring accuracy of the FGS. Finally, a sample of known single and multiple stars will be observed to determine the FGS instrumental transfer function.

\section{Software for HST Data Reduction}

A library of astrometric data reduction software (ADRS) will be available to HST astrometric observers within the science data analysis system (SDAS) at the Space Telescope Science Institute (STScI). The ADRS user will pick from a modular list those software units to be applied to the data. Each module performs its defined operation on the data file, producing a new data file on which 


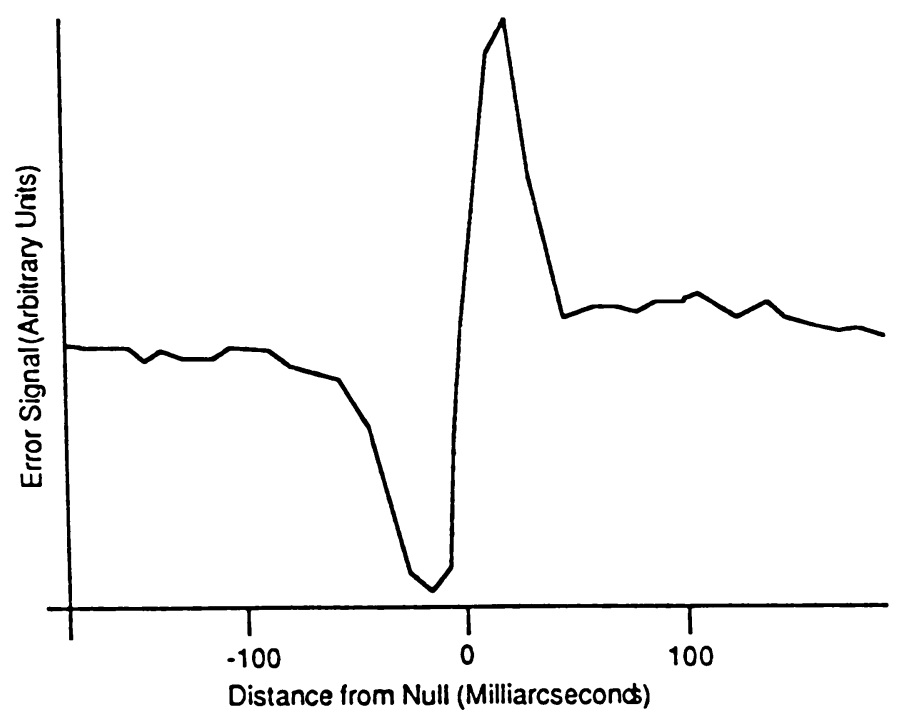

Figure 4. FGS transfer function.

further operations may be performed. The user may select from among standard operations which are available with any operating system (such as, creating and deleting files, editing, copying and combining files, saving and renaming files), and special modules created for astrometric purposes. The computer operating system allows the user to define and name a sequence of operations in which one software unit follows another. An on-line "help" program is provided to assist the users.

Numerous special astrometric software modules have been written specifically for HST data reduction. Included are modules for conversion among various coordinate systems, for the determination of the mean position of an object from a sequence of encoder readings (centroiding), for correction of the data for various instrumental effects (optical distortion, tilt, color terms), and to correct for physical effects such as differential aberration due to vehicle motion as well as the motion of the Earth. A special module for the analysis of transfer function data has been prepared at the Lowell Observatory capable of providing position angle and separation of double star components which have separations greater than about 0:03.

A basic unit of ADRS is GaussFit. This program has its own computer language, which is designed to facilitate the specification of complex data reduction models. The programming language of GaussFit provides a straightforward and easy way to formulate problems in nonlinear estimation, problems with correlated observations, problems where an equation of condition may contain more than one observation, problems involving exact constraints among the parameters, and problems in 
which the model can only be expressed algorithmically and not as a closed form expression. GaussFit uses orthogonal transformations instead of normal equations to solve the basic least squares problem, and it also allows the user to specify a robust estimation method that is resistant to outliers in the data. A GaussFit user's manual is available and further information may be obtained from W. H. Jefferys.

\section{Proposed HST Astrometric Research Programs}

HST astrometry, with accuracies from two to ten times greater than conventional ground based observations, will provide possibilities for new and exciting areas of research.

It will be possible to measure stellar parallaxes with an accuracy of \pm 0 ".001 or better, providing for extension of the trigonometric parallax method to star clusters and RR Lyrae stars. It will also allow improvement in the parallaxes of such fundamental distance calibrators as the Hyades. This improved measurement accuracy will make it possible to investigate systematic errors in parallaxes derived from ground observations. It may become possible to measure the parallaxes of central stars of some planetary nebulae.

In the study of double stars, the separation range of 0.02 to 1.0 is difficult to observe from the ground. Consequently, the statistics of double stars in this range are not well known. Statistics can be improved, however, if a scan of a star in the astrometric FGS is made routinely every time the telescope is pointed at some primary target for observation by one of the other instruments. Parallel observations such as this are feasible but their frequency is an operational matter that remains to be determined. In the case of known spectroscopic binaries, it may be possible to obtain visual orbits, thus providing more information about stellar masses.

It is proposed to utilize HST WFPC observations to detect the motions of stars within clusters, and to measure the effects of tidal forces on the cluster and of mass segregation within the cluster. Proper motions of stars will be detectable in a much shorter time than required for ground-based observations, and it may even be possible to measure proper motions in nearby galaxies.

HST observations of solar system objects will furnish new data on the gravitational fields of the planets and satellites. The accuracy of HST measurement of intersatellite angular distances will provide data good to $5 \mathrm{~km}$ at the distance of Jupiter and $10 \mathrm{~km}$ at the distance of Saturn. Longer term observations by HST of the satellite systems first observed by Voyager will help to solve some of the dynamical questions raised by the short duration data obtained on the Voyager fly-by.

Other proposed research programs include the determination of the gravitational deflection of light by Jupiter, the search for planets of nearby stars and the measurement of optical proper motions in quasars.

Observations with the FGS units of HST will provide a major contribution to fundamental astronomy in a cooperative program with the ESA astrometric satellite HIPPARCOS. The HIPPARCOS project was planned to produce a set of stellar positions, proper motions and parallaxes by essentially observing chords on the celestial sphere. The failure of HIPPARCOS I to achieve its planned orbit may prevent it from obtaining the approximately 100,000 stellar positions and parallaxes with the expected accuracy of \pm 0 ".001. Whatever the accuracy ultimately obtained, the 
reference frame for these data will be the HIPPARCOS Instrumental System which will have an unknown zero point and an unknown "solid body" rotation with respect to a non-rotating reference frame. It remains then, to determine the rotation of the HIPPARCOS Instrumental System and to tie it to some fundamental or absolute reference frame. Because of its capability to measure precise angular distances between objects of disparate magnitude, the HST FGS can be used to tie the HIPPARCOS Instrumental System to (a) very distant and hence relatively motionless objects such as quasars; (b) an absolute coordinate system derived from radio interferometric observations using radio sources which have discrete optical counterparts, and (c) a dynamical system such as that defined by the motions of selected asteroids. With respect to projects (a) and (b) plans have been made to observe the positions and motions of 160 HIPPARCOS catalog stars with respect to 90 extragalactic objects using the HST FGS or in some cases for fainter objects, the WFPC in conjunction with the guiding FGS units. With regard to project (c), plans are made to relate the orbits of selected minor planets, particularly at apparent crossing points, to stars in the HIPPARCOS catalog. Eight thousand candidate stars were selected and of these two thousand were accepted for the HIPPARCOS catalog.

Although HIPPARCOS I may not achieve all of its planned objectives, the HST Astrometry Team intends to carry through all of the planned link observations mentioned in (a), (b) and (c) that can be accommodated within the constraints of the HST ground system. These observations will be linked not only to the observations of HIPPARCOS I but also to on-going ground-based surveys by transit circle observations in both the northern and southern hemispheres.

We feel that the scope and intent of the program planned by the HIPPARCOS Project is of fundamental importance to all of astronomy and we whole-heartedly support the dedicated HIPPARCOS Team in their efforts to realize the ultimate goals of the original HIPPARCOS project.

The HST is now scheduled for launch in the Spring of 1990 . We are eagerly awaiting this event to determine its astrometric capability.

\section{Acknowledgements}

The authors are pleased to acknowledge the cooperation of the HIPPARCOS Input Catalog group at Meudon, colleagues at Lowell Observatory, Georgia State University and Imperial College London for Speckle observations, and at Cambridge and the Astronomisches Rechen-Institut for finding charts.

We wish also to thank our colleagues on the Space Telescope Astrometry Team, Laurence Fredrick, William van Altena and Otto Franz for their cooperation on this project. We gratefully acknowledge support from the National Aeronautics and Space Administration under contract NAS8-32906. 


\section{References}

1. M. Froeschle and J. Kovalevsky, Astron. and Astrophys.,116, 89, 1982.

2. P.D. Hemenway, R.L. Duncombe, W.H. Jefferys and P.J. Shelus, "Using Space Telescope to Tie the HIPPARCOS and Extragalactic Reference Frames Together", in HIPPARCOS: Scientific Aspects of the Input Catalogue Preparation, T.D. Guyenne and J. Hunt, eds., European Space Agency Special Publication ESA SP-234, p.261, 1985.

3. P.D. Hemenway and R.L. Duncombe, "The Use of Space Telescope to Tie the HIPPARCOS Reference Frame to an Extragalactic Reference Frame", in Astrometric Techniques, IAU Symposium \#109, H.K. Eichhorn and R.J. Leacock eds. p. 613, 1986.

4. W.H. Jefferys, G.F. Benedict, P.D. Hemenway, P.J. Shelus and R.L. Duncombe, "Prospects for Astrometry with the Hubble Space Telescope", Celestial Mechanics, 37, 299, 1985.

5. W.H. Jefferys, M.J. Fitzpatrick and B.E. McArthur, "GaussFit: A System for Least Squares and Robust Estimation" Celestial Mechanics, 41, 39, 1988.

\section{Discussion}

KLIONER: You said that the scientific program includes the measurement of the relativistic deflection due to Jupiter. Why only due to Jupiter? For example, the relativistic deflection of the light passing Saturn is 0.006 arcsec. What relativistic corrections do you take into account in your software?

DuncombE: At present the only proposal in the program is the measurement of the relativistic deflection of light due to Jupiter. Other experiments may be proposed later.

STRAND: You mentioned that an accuracy of 0.002 arcsec will be attainable with the Hubble Space Telescope. Is that from a single observation or is it the result of several? Parallaxes of 0.001 arcsec mean error have already been obtained from ground based observations. With respect to investigation of systematic errors of stellar parallaxes, such a program is already in progress at the Flagstaff Statin of the U.S. Naval Observatory by observations of Quasars.

DuncomBE: It is the result of several observations. It should be noted that observational aims of the Hubble Space Telescope, established ten years ago, are now close to realization by groundbased techniques due to the rapid advancements in new technology.

KOPEJKIN: How long should we observe stars in the Large Magellanic Cloud for determination of proper motion of stars in this galaxy?

DuncomBE: The observations should be conducted over three years at least and over five years if possible, depending on the lifetime of the satellite. 